\title{
Логика функций vs логика отношений
}

\author{
В. И. ШАЛАК
}

\begin{abstract}
It is proved that for any first-order theory with equality, the domain of interpretation of which contains at least two individuals, there exists mutually embeddable theory in language with functional symbols and only one-place predicate.
\end{abstract}

Ключевые слова: погружающая операция, функция, предикат

Довольно распространенной является точка зрения, что переход к современной логике, совершенный на рубеже XIX-XX вв., был связан с расширением выразительных возможностей ее языка за счет включения в него суждений об отношениях. Субъектно-предикатная структура предложений традиционной логики не позволяла выразить даже такое простое отношение как «x больше $y »$. С этой проблемой сталкивается Сократ в диалоге Платона «Теэтет».

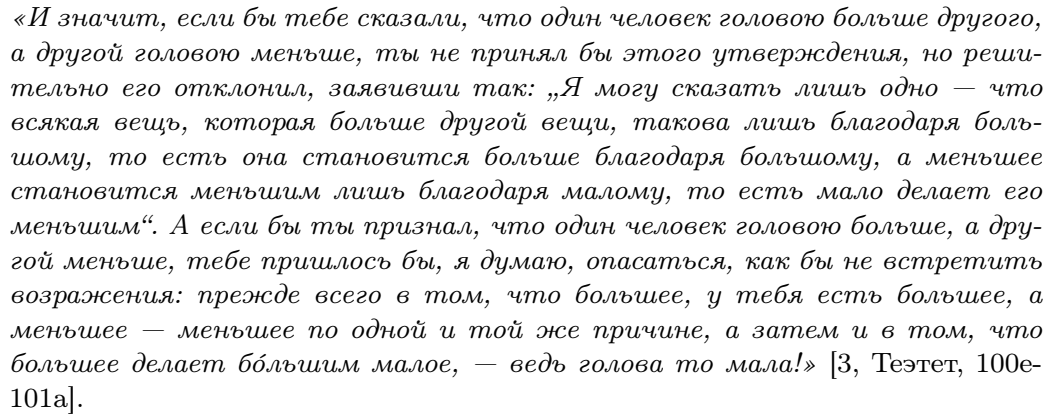

Считается, что переход к логике отношений был закономерным шагом, который благотворно сказался не только на самой логике, но и на общем развитии науки. 


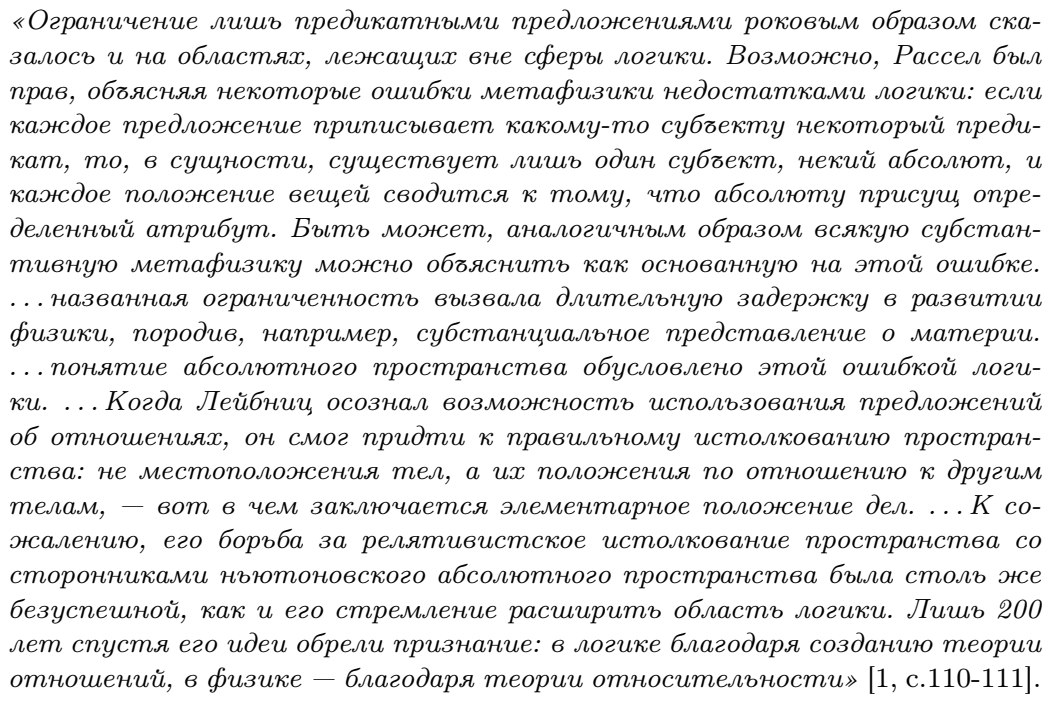

Мы покажем, что вопреки устоявшемуся мнению язык свойств (одноместных отношений) и функций вполне достаточен для выражения тех же математических и физических идей, которые находят оформление в терминах многоместных отношений. С логической точки зрения, не было никакой необходимости для отказа от имевших богатую историю субстантивной метафизики и абсолютного пространства, о чем пишет Р. Карнап.

Напомним некоторые определения, связанные с понятием погружающих операций [4].

Пусть $S$ - теория в языке первого порядка $L$. Принадлежность формулы $A$ языку $L$ будем обозначать посредством $A \in L$, а доказуемость формулы $A$ в теории $S-$ посредством $S \vdash A$. Поскольку теория понимается как дедуктивно замкнутое множество формул, то $S \vdash A$ означает то же самое, что и $A \in S$.

Пусть $S_{1}$ и $S_{2}$ - теории в языках $L_{1}$ и $L_{2}$. Рекурсивную функцию $\varphi: L_{1} \rightarrow L_{2}$ называют операцией, погружающей теорию $S_{1}$ в $S_{2}$, если и только если для нее выполняется следующее условие:

$$
S_{1} \vdash A \Leftrightarrow S_{2} \vdash \varphi(A) .
$$

Будем говорить, что теория $S_{1}$ погружаема в теорию $S_{2}$, если и только если существует операция, погружающая $S_{1}$ в $S_{2}$. 
Теории $S_{1}$ и $S_{2}$ взаимопогружаемы, если и только если $S_{1}$ погружаема в $S_{2}$, и $S_{2}$ погружаема в $S_{1}$. Отношение взаимопогружаемости рефлексивно, симметрично и транзитивно [4, с. 110].

Будем говорить, что теория $S_{1}$ является подтеорией $S_{2}$, если и только если $S_{1} \subseteq S_{2}$.

ЛЕММА 1. Если теория $S_{1}$ является подтеорией $S_{2}$, то достаточным условием взаимопогружаемости $S_{1}$ и $S_{2}$ является существование рекурсивной функции $\varphi: L_{2} \rightarrow L_{1}$, для которой выполняются следующие условия:

1. $S_{2} \vdash A \Rightarrow S_{1} \vdash \varphi(A)$,

2. $S_{1} \vdash \varphi(A) \Rightarrow S_{1} \vdash A \partial \Omega я A \in L_{1}$,

3. $S_{2} \vdash \varphi(A) \Rightarrow S_{2} \vdash A \partial \Omega я A \in L_{2}$.

Доказательство. Операцией, погружающей теорию $S_{1}$ в теорию $S_{2}$, является тождественная функция $\imath(A)=A$.

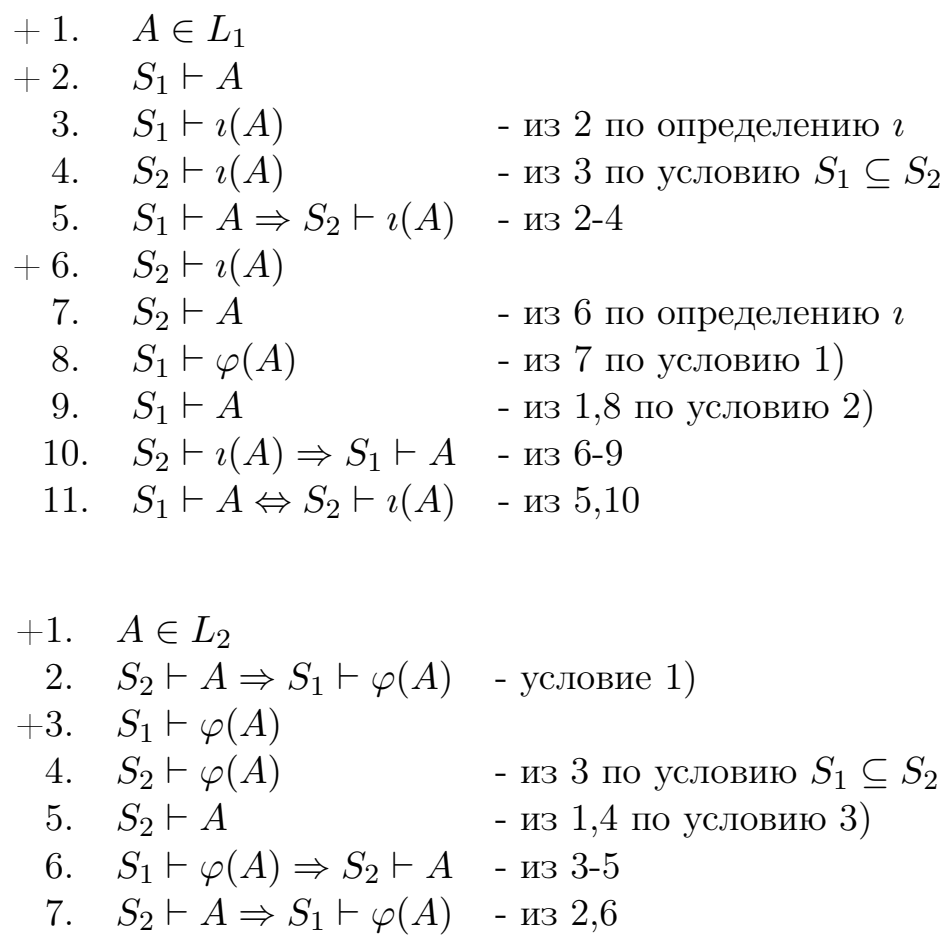


Q.E.D.

Пусть нам дана теория $\mathbf{T}_{\mathbf{1}}$ в языке исчисления предикатов первого порядка с равенством, одной из аксиом которой является формула:

$$
(A x-a b) \quad \neg(a=b),
$$

где $a$ и $b-$ замкнутые термы.

ЛЕММА 2. Если $P-n$-местный предикатный символ языка теории $T_{1}$, то в ней доказуемы следующие формуль:

1. $\exists y((P(\boldsymbol{x}) \& y=a) \vee(\neg P(\boldsymbol{x}) \& y=b))$,

2. $((P(\boldsymbol{x}) \& y=a) \vee(\neg P(\boldsymbol{x}) \& y=b)) \&((P(\boldsymbol{x}) \& z=a) \vee$ $(\neg P(\boldsymbol{x}) \& z=b)) \supset y=z$,

где $\boldsymbol{x}$ - кортеж $n$-переменных.

\section{Доказательство.}

+1 . $P(\boldsymbol{x})$

2. $\vdash a=a$

3. $P(\boldsymbol{x}) \& a=a$

- акс. равенства

4. $(P(\boldsymbol{x}) \& a=a) \vee(\neg P(\boldsymbol{x}) \& a=b)$

- из 1,2

5. $\exists y((P(\boldsymbol{x}) \& y=a) \vee(\neg P(\boldsymbol{x}) \& y=b)) \quad$ - из 4 по $\exists$ в

+6 . $\neg P(\boldsymbol{x})$

7. $\vdash b=b$

8. $\neg P(\boldsymbol{x}) \& b=b$

- акс. равенства

9. $\quad(P(\boldsymbol{x}) \& a=a) \vee(\neg P(\boldsymbol{x}) \& a=b)$

- из 6,7

10. $\exists y((P(\boldsymbol{x}) \& y=a) \vee(\neg P(\boldsymbol{x}) \& y=b)) \quad$ - из 9 по $\exists$ в

11. $T_{1} \vdash y((P(\boldsymbol{x}) \& y=a) \vee(\neg P(\boldsymbol{x}) \&$ $y=b))$

+1. $\quad((P(\boldsymbol{x}) \& y=a) \vee(\neg P(\boldsymbol{x}) \& y=b)) \&$ $((P(\boldsymbol{x}) \& z=a) \vee(\neg P(\boldsymbol{x}) \& z=b))$

2. $(P(\boldsymbol{x}) \& y=a) \vee(\neg P(\boldsymbol{x}) \& y=b) \quad$ - из 1

3. $(P(\boldsymbol{x}) \& z=a) \vee(\neg P(\boldsymbol{x}) \& z=b) \quad$ - из 1

+4 . $\quad P(\boldsymbol{x})$

5. $\quad P(\boldsymbol{x}) \& y=a$ 


$$
\begin{aligned}
\text { 6. } & P(\boldsymbol{x}) \& z=a \\
\text { 7. } & y=a \\
\text { 8. } & z=a \\
9 . & y=z \\
+10 . & \neg P(\boldsymbol{x}) \\
\text { 11. } & \neg P(\boldsymbol{x}) \& y=b \\
\text { 12. } & \neg P(\boldsymbol{x}) \& z=b \\
\text { 13. } & y=b \\
\text { 14. } & z=b \\
\text { 15. } & y=z \\
\text { 16. } & y=z \\
\text { 17. } & T_{1} \vdash((P(\boldsymbol{x}) \& y=a) \vee(\neg P(\boldsymbol{x}) \& \\
& y=b)) \&((P(\boldsymbol{x}) \& z=a) \vee(\neg P(\boldsymbol{x}) \& \\
& z=b)) \supset y=z
\end{aligned}
$$

- из 3,4

- из 5

- из 6

- из 7,8

- из 2,10

- из 3,10

- из 11

- из 12

- из 13,14

- из 4-9, 10-15

- из 1-16

Q.E.D.

Рассмотрим теорию $\mathbf{T}_{\mathbf{2}}$, полученную путем расширения языка теории $T_{1}$ за счет добавления для каждого $n$-местного предикатного символа $P$ нового функционального символа $f_{P}$ и принятия для каждого из них новой аксиомы:

$$
\left(A x-f_{P}\right) \quad\left(P(\boldsymbol{x}) \& f_{P}(\boldsymbol{x})=a\right) \vee\left(\neg P(\boldsymbol{x}) \& f_{P}(\boldsymbol{x})=b\right),
$$

где $\boldsymbol{x}$ - кортеж $n$-переменных.

ЛЕММА 3. В теории $T_{2}$ доказуемъ следующие формуль:

1. $f_{P}(\boldsymbol{x})=a \vee f_{P}(\boldsymbol{x})=b$,

2. $P(\boldsymbol{x}) \equiv f_{P}(\boldsymbol{x})=a$.

\section{Доказательство.}

+1. $\quad P(\boldsymbol{x})$

2. $P(\boldsymbol{x}) \vee\left(f_{P}(\boldsymbol{x})=b\right) \quad$ - из 1

3. $\neg\left(\neg P(\boldsymbol{x}) \& f_{P}(\boldsymbol{x})=b\right) \quad$ - из 2

4. $P(\boldsymbol{x}) \& f_{P}(\boldsymbol{x})=a \quad$ - из 3 и $A x-f_{P}$

5. $f_{P}(\boldsymbol{x})=a \quad$ - из 4

6. $f_{P}(\boldsymbol{x})=a \vee f_{P}(\boldsymbol{x})=b \quad$ из 5 


\begin{tabular}{|c|c|c|}
\hline+7 . & $\neg P(\boldsymbol{x})$ & \\
\hline 8. & $\neg P(\boldsymbol{x}) \vee\left(f_{P}(\boldsymbol{x})=a\right)$ & - из 7 \\
\hline 9. & $\neg\left(P(\boldsymbol{x}) \& f_{P}(\boldsymbol{x})=a\right)$ & - из 8 \\
\hline 10. & $\neg P(\boldsymbol{x}) \& f_{P}(\boldsymbol{x})=b$ & - из 9 и $A x-f_{P}$ \\
\hline 11. & $f_{P}(\boldsymbol{x})=b$ & - из 10 \\
\hline 12. & $f_{P}(\boldsymbol{x})=a \vee f_{P}(\boldsymbol{x})=b$ & - из 11 \\
\hline 13. & $T_{2} \vdash f_{P}(\boldsymbol{x})=a \vee f_{P}(\boldsymbol{x})=b$ & - из 1-6, 7-12 \\
\hline+1 . & $f_{P}(\boldsymbol{x})=a$ & \\
\hline 2. & $\neg\left(f_{P}(\boldsymbol{x})=b\right)$ & - из $1, A x-a b$ \\
\hline 3. & $P(\boldsymbol{x}) \& f_{P}(\boldsymbol{x})=a$ & - из $2, A x-f_{P}$ \\
\hline 4. & $P(\boldsymbol{x})$ & - из 3 \\
\hline 5. & $f_{P}(\boldsymbol{x})=a \supset P(\boldsymbol{x})$ & $-1-4$ \\
\hline+6 & $P(\boldsymbol{x})$ & \\
\hline 7. & $P(\boldsymbol{x}) \& f_{P}(\boldsymbol{x})=a$ & - из $6, A x-f_{P}$ \\
\hline 8. & $f_{P}(\boldsymbol{x})=a$ & - из 7 \\
\hline 9. & $P(\boldsymbol{x}) \supset f_{P}(\boldsymbol{x})=a$ & - из 6-8 \\
\hline 10. & $T_{2} \vdash P(\boldsymbol{x}) \equiv f_{P}(\boldsymbol{x})=a$ & - из 5,9 \\
\hline
\end{tabular}

Q.E.D.

ЛЕММА 4. Для всякой формуль А теории $T_{2}$ существует такая формула $\phi(A)$ языка теории $T_{1}$, что

1. $T_{2} \vdash A \equiv \phi(A)$,

2. $T_{2} \vdash A \Rightarrow T_{1} \vdash \phi(A)$,

3. $T_{2} \vdash A \Rightarrow T_{1} \vdash A-$ если $A$ принадлежит языку теории $T_{1}$.

Эта теорема соответствует предложению 2.29 о введении новых функциональных символов из книги [2]. Условием его применимости является лемма 3 и определение теории $T_{2}$. Поэтому мы можем опустить ее доказательство.

ЛЕММА 5. Теории $T_{1}$ и $T_{2}$ взаимопогружаемы. 
Доказательство. Так как теория $T_{1}$ является подтеорией $T_{2}$, достаточно найти функцию, удовлетворяющую условиям леммы 1.

Пусть $\phi$ - отображение теории $T_{2}$ в теорию $T_{1}$, определяемое в лемме 4 . На основании свойства 2 функции $\phi$ из леммы 4 имеет место $T_{2} \vdash A \Rightarrow T_{1} \vdash \phi(A)$. Проверим выполнимость оставшихся двух условий.

+1. $A \in L_{1}$

$+2 . \quad T_{1} \vdash \phi(A)$

3. $T_{2} \vdash \phi(A) \quad$ - из 2 , так как $T_{1} \subseteq T_{2}$

4. $T_{2} \vdash A \equiv \phi(A) \quad$ - свойство 1 функции $\phi$ из леммы 4

5. $T_{2} \vdash A \quad$ - из 3,4

6. $T_{1} \vdash A \quad$ из 1,5 по свойству 3 из леммы 4

7. $T_{1} \vdash \phi(A) \Rightarrow T_{1} \vdash A \quad$ - из $2-6$

$+1 . \quad A \in L_{2}$

+2 . $\quad T_{2} \vdash \phi(A)$

3. $T_{2} \vdash A \equiv \phi(A) \quad$ - свойство 1 функции $\phi$ из леммы 4

4. $T_{2} \vdash A \quad$ - из 2,3

5. $T_{2} \vdash \phi(A) \Rightarrow T_{2} \vdash A \quad$ - из $2-4$

Q.E.D.

Определим функцию $\alpha$ из языка теории $T_{2}$ в ее подъязык, содержащий лишь функциональные символы и единственный двухместный предикатный символ равенства.

- $\alpha\left(t_{1}=t_{2}\right) \quad=\left(t_{1}=t_{2}\right)$

- $\alpha\left(P\left(t_{1}, \ldots, t_{n}\right)\right)=f_{P}\left(t_{1}, \ldots, t_{n}\right)=a$

- $\alpha(\neg A) \quad=\neg \alpha(A)$

- $\alpha(A \nabla B) \quad=\alpha(A) \nabla \alpha(B) \quad \nabla \in\{\&, \vee, \supset, \equiv\}$

- $\alpha(Q x B) \quad=Q x \alpha(B) \quad Q \in\{\exists, \forall\}$

Пусть формула $A$ является аксиомой теории $\mathbf{T}_{\mathbf{3}}$, если и только если $A=\alpha(B)$, где $B$ собственная аксиома теории $T_{2}$.

ЛЕММА 6. Теории $T_{3}$ и $T_{2}$ взаимопогружаемъ. 
Доказательство. По определению теории $T_{3}$, ее язык является подъязыком теории $T_{2}$.

Если $A$ - аксиома $T_{3}$, то $A=\alpha(B)$, где $B$ собственная аксиома теории $T_{2}$. Так как в силу леммы $3(2)$, определения $\alpha$, и теоремы 2.20 из [2] имеет место $T_{2} \vdash B \equiv \alpha(B)$, то $T_{2} \vdash \alpha(B)$. Отсюда следует, что всякое доказательство в теории $T_{3}$ одновременно является доказательством в $T_{2}$, т.е. $T_{3} \subseteq T_{2}$.

Так как теория $T_{3}$ является подтеорией $T_{2}$, достаточно найти функцию, удовлетворяющую условиям леммы 1.

Индукцией по построению доказательства формулы $A$ в теории $T_{2}$ покажем, что в теории $T_{3}$ доказуема формула $\alpha(A)$.

Если $A$ - логическая аксиома, то $\alpha(A)$ также логическая аксиома и, следовательно, $T_{3} \vdash \alpha(A)$.

Если $A$ - собственная аксиома теории $T_{2}$, то $\alpha(A)$ - аксиома теории $T_{3}$ и потому $T_{3} \vdash \alpha(A)$.

Допустим, формула $A$ получена в теории $T_{2}$ из двух предшествующих формул доказательства $B$ и $(B \supset A)$ по правилу modus ponens. По индуктивному допущению, в теории $T_{3}$ доказуемы формулы $\alpha(B)$ и $\alpha(B \supset A)$. Так как $\alpha(B \supset A)=\alpha(B) \supset$ $\alpha(A)$, то в теории $T_{3}$ доказуема формула $\alpha(A)$.

Допустим, формула $A$ имеет вид $\forall x B$ и получена в теории $T_{2}$ из предшествующей формулы доказательства В по правилу генерализации. По индуктивному допущению, в теории $T_{3}$ доказуема формула $\alpha(B)$. По правилу генерализации в теории $T_{3}$ доказуема формула $\forall x \alpha(B)$. Так как $\forall x \alpha(B)=\alpha(\forall x B)$, то в теории $T_{3}$ доказуема формула $\alpha(A)$.

Таким образом, мы показали, что $T_{2} \vdash A \Rightarrow T_{3} \vdash \alpha(A)$. Проверим выполнимость оставшихся двух условий.

$$
\begin{array}{rll}
+1 . & A \in L_{3} & \\
+2 . & T_{3} \vdash \alpha(A) & \\
\text { 3. } & \alpha(A)=A & \text { - из } 1 \text { пз } 2,3 \\
4 . & T_{3} \vdash A & \\
5 . & T_{3} \vdash \alpha(A) \Rightarrow T_{3} \vdash A & \text { - из } 2-4 \\
& & \\
+1 . & A \in L_{2} & \\
+2 . & T_{2} \vdash \alpha(A) & \text { - в силу леммелению } \alpha \\
\text { 3. } & T_{2} \vdash A \equiv \alpha(A) & \text { теоремы } 2.20[2]
\end{array}
$$




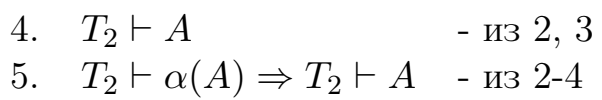

Q.E.D.

Следующая теорема получается как простое следствие ранее доказанных лемм 5 и 6.

TEOPEMA 7. Для всякой первопорядковой теории в языке логики предикатов с равенством, в которой для некоторых двух замкнутых термов а и $b$ доказуема формула $\neg(a=b)$, существует взаимопогрузимая с ней первопорядковая теория в языке с одними лишь функииональными символами и единственным предикатом равенства.

Далее мы покажем, что предикат равенства также не является необходимым и может быть заменен специальным одноместным предикатом. Пусть теория $\mathbf{T}_{\mathbf{4}}$, получена путем расширения языка теории $T_{3}$ новым одноместным предикатным символом $H$ и добавлением новой аксиомы:

$$
(A x-H) \quad(H(x) \equiv x=a) .
$$

ЛЕММА 8. $B$ теории $T_{4}$ доказуема формула $H\left(f_{=}\left(x_{1}, x_{2}\right)\right) \equiv$ $\left(x_{1}=x_{2}\right)$.

\section{Доказательство.}

1. $T_{2} \vdash\left(x_{1}=x_{2}\right) \equiv\left(f_{=}\left(x_{1}, x_{2}\right)=a\right) \quad$ - лемма $3(2)$

2. $T_{2} \vdash A \Rightarrow T_{3} \vdash \alpha(A) \quad$ - лемма 6

3. $T_{3} \vdash\left(x_{1}=x_{2}\right) \equiv\left(f_{=}\left(x_{1}, x_{2}\right)=a\right) \quad$ - из 1,2 и

4. $T_{3} \subseteq T_{4} \quad$ - определение $T_{4}$

5. $T_{4} \vdash\left(x_{1}=x_{2}\right) \equiv\left(f_{=}\left(x_{1}, x_{2}\right)=a\right) \quad$ - из 3,4

6. $\forall x(H(x) \equiv x=a) \quad$ - аксиома $T_{4}$

7. $T_{4} \vdash H\left(f_{=}\left(x_{1}, x_{2}\right)\right) \equiv\left(f_{=}\left(x_{1}, x_{2}\right)=a\right) \quad$ - из 6

8. $T_{4} \vdash H\left(f_{=}\left(x_{1}, x_{2}\right)\right) \equiv\left(x_{1}=x_{2}\right) \quad$ - из 5,7 
ЛЕММА 9. Теории $T_{3}$ и $T_{4}$ взаимопогружаемы.

Доказательство. Так как теория $T_{3}$ является подтеорией $T_{4}$, достаточно найти функцию, удовлетворяющую условиям леммы 1.

Определим функцию $\psi$ из языка теории $T_{4}$ в язык теории $T_{3}$.

- $\psi(H(t))=t=a$

- $\psi\left(t_{1}=t_{2}\right)=t_{1}=t_{2}$

- $\psi(\neg A)=\neg \psi(A)$

- $\psi(A \nabla B)=\psi(A) \nabla \psi(B) \quad \nabla \in\{\&, \vee, \supset, \equiv\}$

- $\psi(Q x B) \quad=Q x \psi(B) \quad Q \in\{\exists, \forall\}$

Индукцией по построению доказательства формулы $A$ в теории $T_{4}$ покажем, что в этом случае в теории $T_{3}$ доказуема формула $\psi(A)$.

Если $A-$ логическая аксиома, то легко проверить, что $\psi(A)$ также является логической аксиомой и потому $T_{3} \vdash \psi(A)$.

Если $A$ - аксиома $H(x) \equiv(x=a)$, то $\psi(A)$ есть $(x=a) \equiv$ $(x=a)$ и, следовательно, $T_{3} \vdash \psi(A)$.

Если $A-$ собственная аксиома теории $T_{3}$, то $\psi(A)=A$, т.к. $A$ не содержит вхождений предикатного символа $H$. Поэтому $T_{3} \vdash \psi(A)$.

Допустим, формула $A$ получена из двух предшествующих формул доказательства $B$ и $(B \supset A)$ по правилу modus ponens. По индуктивному допущению, в теории $T_{3}$ доказуемы формулы $\psi(B)$ и $\psi(B \supset A)$. Так как $\psi(B \supset A)=\psi(B) \supset \psi(A)$, то в теории $T_{3}$ доказуема формула $\psi(A)$.

Допустим, формула $A$ имеет вид $\forall x B$ и получена из предшествующей формулы доказательства В по правилу генерализации. По индуктивному допущению, в теории $T_{3}$ доказуема формула $\psi(B)$. По правилу генерализации в теории $T_{3}$ доказуема формула $\forall x \psi(B)$. Так как $\forall x \psi(B)=\psi(\forall x B)$, то в теории $T_{3}$ доказуема формула $\psi(A)$.

Таким образом, $T_{4} \vdash A \Rightarrow T_{3} \vdash \psi(A)$. Проверим выполнимость оставшихся двух условий.

+1. $A \in L_{3}$

$+2 . \quad T_{3} \vdash \psi(A)$ 


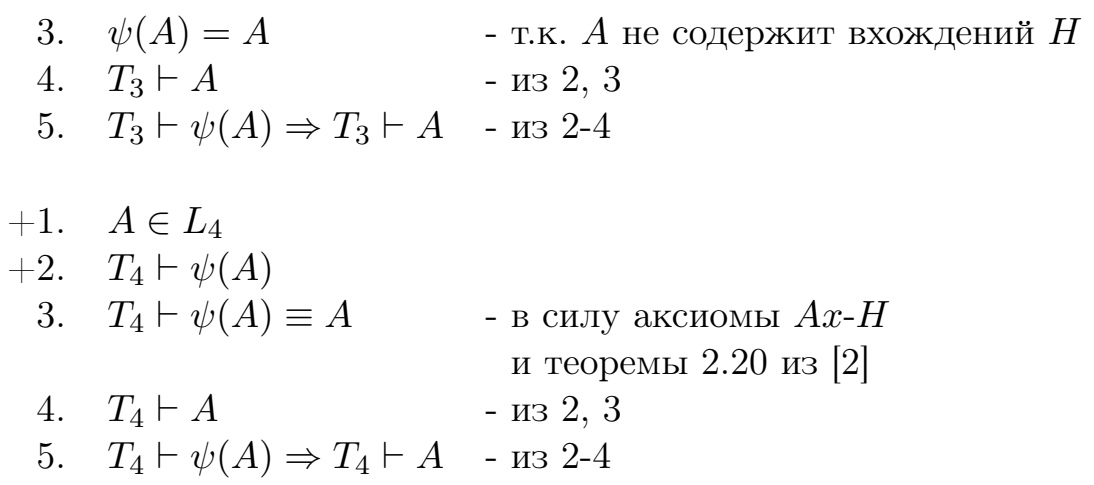

Q.E.D.

Определим функцию $\beta$ из языка теории $T_{4}$ в ее подъязык, содержащий лишь функциональные символы и единственный одноместный предикатный символ $H$.

- $\beta(H(t))=H(t)$

- $\beta\left(t_{1}=t_{2}\right)=H\left(f_{=}\left(t_{1}=t_{2}\right)\right)$

- $\beta(\neg A) \quad=\quad \neg \beta(A)$

- $\beta(A \nabla B) \quad=\beta(A) \nabla \beta(B) \quad \nabla \in\{\&, \vee, \supset, \equiv\}$

- $\beta(Q x B)=Q x \beta(B) \quad Q \in\{\exists, \forall\}$

Пусть формула $A$ является аксиомой теории $\mathbf{T}_{\mathbf{5}}$, если и только если $A=\beta(B)$, где $B$ либо аксиома равенства, либо собственная аксиома теории $T_{4}$.

ЛЕММА 10. Теории $T_{5}$ и $T_{4}$ взаимопогружаемы.

Доказательство. По определению теории $T_{5}$ ее язык является подъязыком теории $T_{4}$.

Если $A$ - аксиома $T_{5}$, то $A=\beta(B)$, где $B$ либо аксиома равенства, либо собственная аксиома теории $T_{4}$. В силу леммы 8 , определения $\beta$ и теоремы 2.20 из [2] имеет место $T_{4} \vdash B \equiv \beta(B)$ и, следовательно, $T_{4} \vdash \beta(B)$. Т.е. всякая аксиома теории $T_{5}$ доказуема в $T_{4}$. Отсюда получаем, что всякое доказательство в теории $T_{5}$ одновременно является доказательством в $T_{4}$, т.е. $T_{5} \subseteq T_{4}$.

Так как теория $T_{5}$ является подтеорией $T_{4}$, достаточно найти функцию, удовлетворяющую условиям леммы 1. 
Индукцией по построению доказательства формулы $A$ в теории $T_{4}$ покажем, что в этом случае в теории $T_{5}$ доказуема формула $\beta(A)$.

Если $A$ - пропозициональная или кванторная аксиома, то $\beta(A)$ также пропозициональная или кванторная аксиома и, следовательно, $T_{5} \vdash \beta(A)$.

Если $A$ - либо аксиома равенства, либо собственная аксиома теории $T_{4}$, то $\beta(A)-$ аксиома теории $T_{5}$ и потому $T_{5} \vdash \beta(A)$.

Допустим, формула $A$ получена в теории $T_{4}$ из двух предшествующих формул доказательства $B$ и $(B \supset A)$ по правилу modus ponens. По индуктивному допущению, в теории $T_{5}$ доказуемы формулы $\beta(B)$ и $\beta(B \supset A)$. Так как $\beta(B \supset A)=\beta(B) \supset$ $\beta(A)$, то в теории $T_{5}$ доказуема формула $\beta(A)$.

Допустим, формула $A$ имеет вид $\forall x B$ и получена в теории $T_{4}$ из предшествующей формулы доказательства В по правилу генерализации. По индуктивному допущению, в теории $T_{5}$ доказуема формула $\beta(B)$. По правилу генерализации в теории $T_{5}$ доказуема формула $\forall x \beta(B)$. Так как $\forall x \beta(B)=\beta(\forall x B)$, то в теории $T_{5}$ доказуема формула $\beta(A)$.

Таким образом, мы показали, что $T_{4} \vdash A \Rightarrow T_{5} \vdash \alpha(A)$. Проверим выполнимость оставшихся двух условий.

$$
\begin{aligned}
& +1 . \quad A \in L_{5} \\
& +2 \text {. } \quad T_{5} \vdash \beta(A) \\
& \text { 3. } \beta(A)=A \quad \text { - из } 1 \text { по определению } \beta \\
& \text { 4. } T_{5} \vdash A \quad \text { - из } 2,3 \\
& \text { 5. } \quad T_{5} \vdash \beta(A) \Rightarrow T_{5} \vdash A \quad \text { - из 2-4 } \\
& \text { +1. } A \in L_{4} \\
& +2 \text {. } \quad T_{4} \vdash \beta(A) \\
& \text { 3. } T_{4} \vdash A \equiv \beta(A) \quad \text { - в силу леммы } 8 \text { и } \\
& \text { теоремы } 2.20 \text { [2] } \\
& \text { 4. } T_{4} \vdash A \quad \text { - из } 2,3 \\
& \text { 5. } T_{4} \vdash \beta(A) \Rightarrow T_{4} \vdash A \quad \text { - из } 2-4
\end{aligned}
$$

Q.E.D.

Следующая теорема является простым следствие теоремы 7 и лемм 9 и 10. 
TEOPEMA 11. Для всякой первопорядковой теории в языке логики предикатов с равенством, в которой для некоторых двух замкнутых термов а и $b$ доказуема формула $\neg(a=b)$, существует взаимопогрузимая с ней теория, язык которой содержит лишь функциональные символь и единственный одноместнъй предикат.

Доказательство представленных теоремы не является сложным, и потому их содержание кажется тривиальным. Однако с философской точки зрения они достаточно интересны, поскольку опровергают некоторые устоявшиеся мнения.

\begin{abstract}
«Рассел указал на то, что роковая ошибка школьной философии заключалась в предположении, будто каждое суждение некоторому субгекту приписъвает какое-то свойство в качестве предиката. Если, например, говорят, что тело А движется относительно некоторого другого тела $B$, то представитель школьной логики будет требоватъ, чтобы одному или другому телу самому по себе был приписан предикат движения. Рассел показал, что оченъ многие суждения говорят об отношении, о связи двух обгектов, и их нельзя свести к высказываниям о присущности свойства отдельному объекту. Последние представляют собой лишъ частный случай высказываний об отношениях. Поэтому представителю школъной логики кажутся бессмысленными высказывания, например, такого вида: если два тела движутся относительно друг друга, то не имеет никакого смысла спрашиватъ, какое из них „действителъно движется“, т.е. какому из них присущ, предикат „находиться в движении“» [5, с.172].
\end{abstract}

Рассел ошибался, когда думал, будто показал, что суждения об отношениях нельзя свести к высказываниям о присущности свойства отдельному объекту. Можно предположить, что в результате этой ошибки наша картина мира оказалась искусственно искаженной.

\title{
Литература
}

[1] Карнап P. Старая и новая логика // Журнал «Erkenntnis» («Познание»). Избранное. М.: Издательский дом «Территория будущего», Идея-Пресс, 2007. C. $105-119$.

[2] Мендельсон Э. Введение в математическую логику. М.: Наука, 1976.

[3] Платон. Собрание сочинений в 4 т. М.: Мысль, 1993.

[4] Смирнов B.A. Логические методы анализа научного знания. М.: Наука, 1987.

[5] Франк Ф. Каково значение современных физических теорий для общей теории познания? // ЖКурнал «Erkenntnis» («Познание»). Избранное. М.: Издательский дом «Территория будущего», Идея-Пресс, 2007. С. 160-187. 